\title{
Cellulose nanocrystals with tethered polymer chains: chemically patchy versus uniform decoration
}

\author{
Justin O. Zoppe, $\| \dagger *$ Alix Vaimiti Marie Dupire, $\|$ Théo Gaston Gérard Lachat, $\|$ Philipp Lemal, ${ }^{\ddagger}$ Laura \\ Rodriguez-Lorenzo, ${ }^{\ddagger}$ Alke Petri-Fink, ${ }^{\ddagger}$ Christoph Weder ${ }^{\ddagger}$ and Harm-Anton Klok\|
}

"Ecole Polytechnique Fédérale de Lausanne (EPFL), Institut des Matériaux and Institut des Sciences et Ingénierie Chimiques, Laboratoire des Polymères, Bâtiment MXD, Station 12, CH-1015 Lausanne, Switzerland

${ }^{\ddagger}$ Adolphe Merkle Institute, University of Fribourg, CH-1700 Fribourg, Switzerland

KEYWORDS: cellulose nanocrystals, polymer-tethered nanorods, surface-initiated atom transfer radical polymerization, reducing end groups, colloidal liquid crystals, nanohybrids

\begin{abstract}
The site-specific surface modification of colloidal substrates, yielding "patchy" nanoparticles, is a rapidly expanding area of research as a result of the new complex structural hierarchies that are becoming accessible to chemists and materials scientists through colloidal self-assembly. The inherent directionality of cellulose chains, which feature a non-reducing and a reducing end, within individual cellulose nanocrystals (CNCs) renders them an interesting experimental platform for the synthesis of asymmetric nanorods with end-tethered polymer chains. Here, we present water-tolerant reaction pathways toward patchy and uniformly modified CNC hybrids based on atom transfer radical polymerization (ATRP) and initiators that were linked to the $\mathrm{CNCs}$ with carbodiimide-mediated coupling and Fischer esterification, respectively. Various monomers, including $N$ isopropylacrylamide (NIPAM), [2-(methacryloyloxy)ethyl]trimethylammonium chloride (METAC), and sodium 4vinylbenzenesulfonate (4-SS) were polymerized from both types of initiator-modified CNCs, yielding chemically patchy and uniform CNC hybrids, via surface-initiated ATRP (SI-ATRP). Interestingly, the stereochemistry of tethered PNIPAM was affected by the precise location of ATRP initiating sites, as evidenced by ${ }^{1} \mathrm{H}$ NMR and circular dichroism (CD) spectroscopy. This effect may be related to the inherent right-handed chirality of CNCs. CNC/PMETAC hybrids were labeled with gold nanoparticles (AuNPs) in order to visualize the precise location of polymer tethers via cryo-electron microscopy. In some instances, the AuNPs were indeed concentrated at the end groups of the patchy CNC hybrids.
\end{abstract}

The site-specific surface modification of colloidal substrates, yielding "patchy" (nano)particles, is receiving rapidly expanding interest, as a result of the new complex structural hierarchies that are becoming accessible to chemists and materials scientists through colloidal self-assembly. ${ }^{1}$ Such patchy particles include Janus colloids, ${ }^{2}$ genetically engineered bacteriophages, ${ }^{3,4}$ cylindrical block copolymer micelles, ${ }^{5}$ and polymer-tethered nanorods. ${ }^{6}$ Particle shape anisotropy provides additional building block parameters, which can broaden the complexity of their assemblies. ${ }^{7}$ The lyotropic liquid crystal formation capability of rod-shaped colloidal building blocks is an excellent example of such a self-assembly process. $^{8}$ In special cases, these liquid crystal (LC) phases exhibit longrange chirality, displaying so-called cholesteric or chiral nematic arrangements, which are not only fundamentally intriguing for condensed matter investigations, ${ }^{9}$ but also useful as chiral building blocks for the creation of advanced functional materials. ${ }^{10}$ Relatively few anisotropic colloids that exhibit such chiral phases are known; they include filamentous viruses, ${ }^{11}$ collagen, ${ }^{12}$ chitin, ${ }^{13}$ and cellulose nanocrystals (CNCs).${ }^{14} \mathrm{CNCs}$ are a unique class of colloidal liquid crystals in that their cholesteric LC phases are preserved upon evaporation-induced self-assembly (EISA), which together with their easy accessibility makes them very useful as templates for longrange chiral order in solid-state materials. ${ }^{15}$ One drawback is that the stability of CNC LC phases is sensitive to additives and mostly limited to aqueous media. Polymer grafting at the surface of CNCs has been utilized to make their LC phases more robust ${ }^{16,17}$ however there are few investigations of the EISA process of such hybrid CNC materials. It can also be argued that uniform surface functionalization of $\mathrm{CNCs}$ with tethered polymers disturbs their packing density. ${ }^{18}$ In this context, confining polymer tethers to the ends of the CNCs may present an interesting alternative. The inherent directionality of cellulose chains, i.e. non-reducing end vs. reducing end, within individual $\mathrm{CNCs}^{19}$ makes them an interesting experimental platform for the synthesis and assembly of nanorods with endtethered polymer chains, especially those with tethers at only one end. ${ }^{20}$ Moreover, surface-initiated controlled radical polymerization (SI-CRP) techniques allow precise control of molecular weight and dispersity of tethered polymers, so that the nanorod:tether length ratio can be easily manipulated. ${ }^{21}$ To this end, new synthetic protocols are required that allow attachment of CRP initiators to reducing end groups of CNCs for subsequent SI-CRP and comparisons to uniformly modified CNCs.

In this communication, we present a water-tolerant reaction pathway toward nanorods with polymer chains tethered to the ends of commercially available CNCs by taking advantage of the precise location of reducing end groups. ${ }^{19,22,23}$ To the best of our knowledge, there is only one report on polymer grafting confined to the reducing end groups of $\mathrm{CNCs}$, in which a variety of polymers were either grafted to phenylcarboxylatefunctionalized reducing ends or grafted by free radical 
polymerization (FRP) from reducing ends that had been modified with azo initiators. ${ }^{24}$

(A)
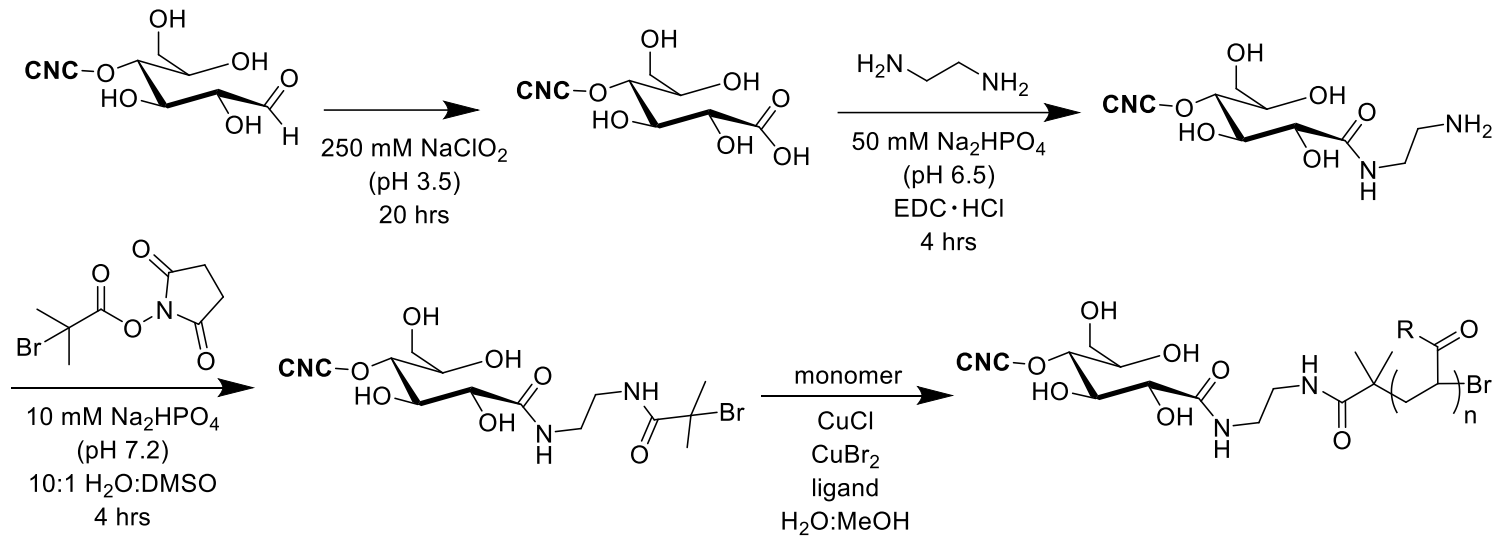

(B)

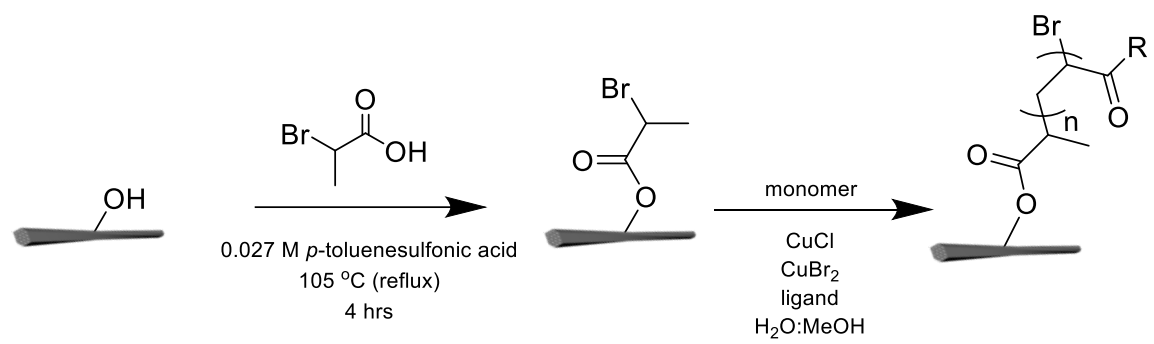

Scheme 1. (A) Synthetic protocols for conducting SI-ATRP from reducing end groups of CNCs ${ }^{23,25}$ and (B) for conducting SIATRP from CNC hydroxyl groups via water tolerant initiator immobilization based on Fischer esterification. ${ }^{26}$

Building on this work, we sought to establish a versatile and water-tolerant protocol to graft polymers from $\mathrm{CNC}$ reducing ends through controlled polymerization techniques, as exemplarily summarized in Scheme 1A. At first, CNC reducing end groups were oxidized and modified with ethylenediamine (EDA), followed by attachment of 2-bromoisobutanoic acid $N$ hydroxysuccinimide ester (NHS-BiB). ${ }^{25}$ For comparison purposes, a CNC sample was prepared in which the surface hydroxyl groups were uniformly modified via $p$-toluenesulfonic acid-catalyzed esterification of 2-bromopropionic acid under reflux conditions that were inspired by previous works (Scheme 1B). ${ }^{26,27}$ Subsequently, various monomers, including $\mathrm{N}$ isopropylacrylamide (NIPAM), [2-(methacryloyloxy)ethyl]trimethylammonium chloride (METAC) and sodium 4vinylbenzenesulfonate (4-SS) were polymerized from both types of initiator-modified CNCs, i.e. chemically "patchy" vs. uniform $\mathrm{CNCs}$, via surface-initiated atom transfer radical polymerization (SI-ATRP).

Wood pulp-derived CNCs obtained from the USDA Forest Products Laboratory (FPL) (supplied by the University of Maine) were first fully characterized in terms of average aspect ratio, surface charge density, and specific surface area. The dimensions of CNCs as received were determined to be $150 \pm$ $26 \mathrm{~nm}$ in length and $5.6 \pm 2.3 \mathrm{~nm}$ in width by AFM, resulting in an average aspect ratio of 27 . The surface charge density was found to be $0.208 \pm 0.009 \mathrm{mmol} / \mathrm{g}$ from a series of conductometric titrations. To ensure full protonation of the CNC's surface sulfate half-ester groups, they were passed through a strong acid cation exchange resin prior to conductometric titration experiments. ${ }^{28}$ Considering the specific surface area of $258 \mathrm{~m}^{2} / \mathrm{g}$ determined from Congo red dye adsorption experiments ${ }^{29}$ (see Supporting Information), the surface charge density was calculated to be $0.485 \mathrm{e} / \mathrm{nm}^{2}$. The surface chemical properties and morphology of the CNCs fell within the range of values recently reported by Reid et al. ${ }^{30}$ The authors also reported that CNCs produced by the FPL contain nearly equal amounts of cellulose I and cellulose II polymorphs. Since cellulose II crystals contain, in addition to parallel arrangements, also anti-parallel chains, reducing end groups may be present on either one or both ends of CNCs. ${ }^{19}$

Following the immobilization of ATRP initiators, either uniformly (2BPA-CNCs) or at the reducing end groups of the $\mathrm{CNCs}$ only (NHSBiB-CNCs), purification by dialysis and lyophilization, the samples were subjected to transmission FTIR and XPS analysis. Figure S3 shows the FTIR spectra of unmodified CNCs in comparison to 2BPA-CNCs and NHSBiBCNCs. IR spectra of the uniformly modified CNCs display the characteristic ester stretch at $1732 \mathrm{~cm}^{-1}$, whereas the corresponding NHSBiB-CNCs spectrum is similar to that of unmodified CNCs. Due to the small expected number of amide bonds generated by initiator attachment to reducing ends of CNCs, this result is unsurprising. The results of XPS experiments also confirmed the higher surface concentration of initiators on 2BPA-CNCs versus NHSBiB-CNCs, as indicated by the higher bromine content shown in Table 1. A decrease in sulfur content was also observed, which was likely a result of hydrolytic desulfation of sulfate half-ester groups under acidic conditions. ${ }^{31}$ As a first case study, PNIPAM was grafted from both types of initiator-modified $\mathrm{CNCs}$ under identical polymerization conditions (experimental details can be found in Supporting Information). Successful grafting of PNIPAM from 2BPA-CNCs and NHSBiB-CNCs was confirmed by TGA and XPS. Shown in Figure 1 are $1^{\text {st }}$ derivative thermogram (DTG) curves of CNC/PNIPAM hybrids along with the traces of unmodified and initiator-modified CNCs. Unmodified CNCs 
showed the characteristic peak of thermal degradation at about $300{ }^{\circ} \mathrm{C}$. 
Table 1. Elemental compositions (\%) and elemental ratios of CNCs and hybrid samples determined by XPS.

\begin{tabular}{|c|c|c|c|c|c|c|c|c|c|c|c|}
\hline Sample & $\begin{array}{l}\text { C 1s } \\
(\%)\end{array}$ & $\begin{array}{l}\text { O 1s } \\
(\%)\end{array}$ & $\begin{array}{l}\text { N 1s } \\
(\%)\end{array}$ & $\begin{array}{c}\mathrm{Na} 1 \mathrm{~s} \\
(\%)\end{array}$ & $\begin{array}{l}\text { S 2p } \\
(\%)\end{array}$ & $\begin{array}{c}\text { Cl 2p } \\
(\%)\end{array}$ & $\begin{array}{c}\mathrm{Br} 3 d \\
(\%)\end{array}$ & $\mathrm{O} / \mathrm{C}$ & $\begin{array}{l}\text { S/O } \\
(\%)\end{array}$ & $\begin{array}{l}\text { N/O } \\
(\%)\end{array}$ & $\begin{array}{c}\text { Cellulose } \\
(\%)\end{array}$ \\
\hline unmodified CNC & 57.03 & 40.77 & 0.41 & 1.18 & 0.47 & 0.05 & 0.09 & 0.71 & 1.2 & 1.0 & - \\
\hline 2BPA-CNC & 56.49 & 42.68 & 0.46 & - & 0.15 & - & 0.23 & 0.76 & 0.35 & 1.1 & - \\
\hline NHSBiB-CNC & 55.49 & 43.43 & 0.27 & 0.47 & 0.32 & 0.02 & 0.01 & 0.78 & 0.74 & 0.62 & - \\
\hline CNC-g-PNIPAM & 72.91 & 16.02 & 10.60 & 0.33 & 0.07 & - & 0.06 & 0.22 & 0.44 & 66 & 25 \\
\hline CNC-RE-g-PNIPAM & 69.30 & 21.82 & 8.55 & - & 0.29 & - & 0.05 & 0.31 & 1.3 & 39 & 62 \\
\hline CNC-g-PMETAC & 66.88 & 26.20 & 4.20 & 0.06 & 0.25 & 2.37 & 0.04 & 0.39 & 0.95 & 16 & 66 \\
\hline CNC-RE-g-PMETAC & 60.42 & 36.12 & 1.98 & 0.38 & 0.45 & 0.66 & - & 0.60 & 1.2 & 5.5 & 75 \\
\hline CNC-g-PSS & 67.28 & 24.86 & 1.45 & 0.55 & 5.79 & - & 0.07 & 0.37 & 23 & 5.8 & 11 \\
\hline CNC-RE-g-PSS & 55.90 & 41.77 & 0.88 & 0.03 & 1.40 & - & 0.03 & 0.75 & 3.4 & 2.1 & 69 \\
\hline
\end{tabular}

By contrast, the DTG trace of 2BPA-CNCs shows two peaks that indicate two different decomposition events. The lower onset temperature is interpreted to result from in-situ acid formation that catalyzes thermal degradation. NHSBiB-CNCs show the same onset temperature, however with a single peak. In the case of the CNCs uniformly decorated with PNIPAM (CNC-g-PNIPAM), the thermal stability is increased to around $350{ }^{\circ} \mathrm{C}$, as a result of the covalently-attached polymer coating. Moreover, the typical peak associated with the degradation of PNIPAM at $400{ }^{\circ} \mathrm{C}$ can be observed..$^{32}$ By contrast, the DTG trace of patchy CNC/PNIPAM hybrids (CNC-RE-g-PNIPAM) displays two separate peaks, similar to what one might expect with a blend of the two components. The elemental composition derived from XPS (Table 1) reveals a higher nitrogen content for uniform CNC/PNIPAM hybrids and less than half the overall cellulose content compared to the patchy $\mathrm{CNC}$ hybrids. All of the above suggested that the surface concentration PNIPAM grafts were indeed affected by the different initiator immobilization chemistries used, however direct visualization was necessary to establish polymer growth confinement to $\mathrm{CNC}$ reducing end groups (vide infra).

In addition to PNIPAM, cationic PMETAC and anionic PSS were grafted from both types of initiator-modified CNCs. The surface elemental compositions of these products are also compiled in Table 1. As for the CNC/PNIPAM hybrids, CNC/PMETAC hybrids show a similar trend in nitrogen content, albeit with overall lower quantities of polymer grafts. This can be explained by the higher rate of propagation of acrylamides compared to methacrylates, as well as the differences in activity of bpy compared to PMDETA ligands. ${ }^{33}$ The XPS results were further correlated with elemental analysis, shown in Table S1. Regarding CNC/PSS hybrids, a stark contrast can be observed between patchy and uniformly grafted materials. Specifically, uniform CNC/PSS hybrids featured an overall cellulose content of just $11 \%$, whereas the patchy hybrids contained still nearly $70 \%$ cellulose, as determined by XPS. The presence of PSS on the patchy hybrids was indeed confirmed by FTIR spectroscopy, shown in Figure $\mathrm{S} 4$. This difference is interpreted as a result of electrostatic repulsion between NHSBiB-CNCs and 4-SS during polymerization, an effect that is less pronounced for 2BPACNCs, according to the trends in sulfur content, i.e. sulfate halfesters, as shown in Table 1. The DTG curves of CNC/PSS and CNC/PMETAC hybrids are shown in Figure S5 and S6, respectively.

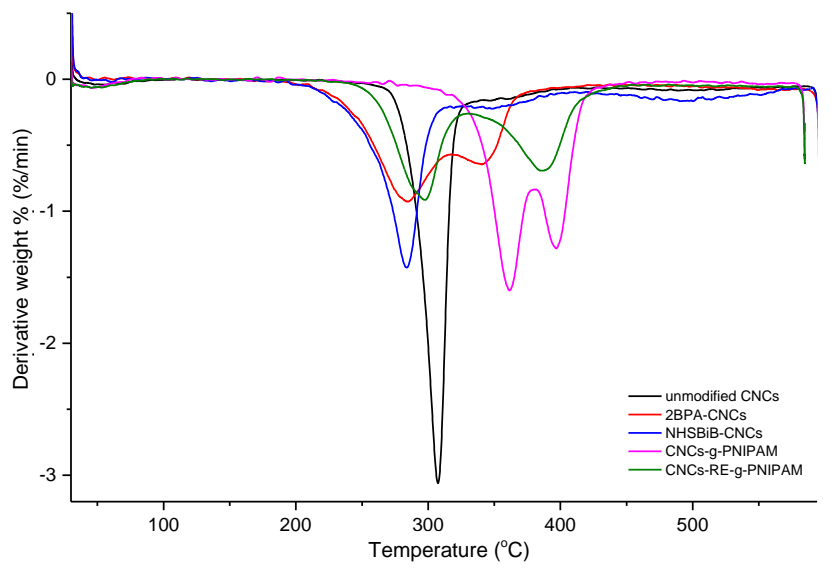

Figure 1. $1^{\text {st }}$ derivative thermogram (DTG) curves of unmodified CNCs (black), 2BPA-CNCs (red), NHSBiB-CNCs (blue), uniform (magenta) and patchy (green) CNC/PNIPAM hybrids.

In general, polymer grafts covalently attached to cellulose via ester bonds can be cleaved by alkaline hydrolysis. ${ }^{21}$ Thus, we attempted to cleave both PNIPAM and PMETAC grafts from uniform and patchy $\mathrm{CNC}$ hybrids by treatment with $2 \% \mathrm{NaOH}$ for $48 \mathrm{~h}$ at room temperature. ${ }^{32}$ We suspected the rate of hydrolysis would be different between uniform and patchy hybrids due to the difference in the polymer tether linkages, i.e. ester vs. amide, respectively. Regardless, we expected to obtain sufficient amounts of cleaved polymer for patchy PNIPAM and PMETAC hybrids based on the results of XPS (Table 1). To address concerns regarding the potential hydrolysis of METAC side chains, the ${ }^{1} \mathrm{H}$ NMR spectra of patchy CNC/PMETAC hybrids were compared to those of PMETAC cleaved from uniform hybrids via alkaline hydrolysis. Gratifyingly, the comparison (Figure S7) confirms the stability of the METAC side chains. The CNC/PSS hybrids were instead subjected to concentrated $\mathrm{H}_{2} \mathrm{SO}_{4}$ at $60{ }^{\circ} \mathrm{C}$ for $3 \mathrm{~h}$ in order to completely hydrolyze CNCs and collect cleaved PSS grafts. Figure S8 shows the ${ }^{1} \mathrm{H}$ NMR spectra of CNC/PSS hybrids and PSS cleaved from uniform hybrids via $\mathrm{H}_{2} \mathrm{SO}_{4}$ hydrolysis. The PSS side chains appeared unaffected by the cleaving conditions, however very low signal intensities for patchy CNC/PSS hybrids were observed.

The inherent right-handed chirality of $\mathrm{CNCs}^{34-36}$ led us to question whether this specific particle propensity would have an effect on the tacticity of surface-grown polymers, especially 
when initiated from the reducing ends. Previous investigations have demonstrated the chain-growth polymerization of stereoregular PNIPAM $^{37}$ and poly(methyl methacrylate) (PMMA $)^{38}$ brushes with coordinating Lewis acids. ${ }^{39}$ Moreover, optically active (isotactic) poly(methacrylic acid) (PMAA) was synthesized using chitosan as a chiral template. ${ }^{40}$ The analysis of the tacticity of PSS and PMETAC based on ${ }^{1} \mathrm{H}$ NMR spectra is non-trivial, however the stereoregularity of PNIPAM is more easily determined. ${ }^{37}$ Figure 2 shows ${ }^{1} \mathrm{H}$ NMR spectra of PNIPAM cleaved from both uniform and patchy CNC/PNIPAM hybrids and free PNIPAM synthesized via solution ATRP, either in the presence or absence of unmodified CNCs. The signals observed at chemical shifts between 1.25 and $2.0 \mathrm{ppm}$ are associated with the $-\mathrm{CH}_{2}$ - groups in the polymer backbone and were used to determine the ratio of meso- to racemo-diads $(\mathrm{m} / \mathrm{r})$ and thus tacticity. ${ }^{37}$ Free PNIPAM synthesized in the absence of unmodified CNCs had an $m / r=$ 49/51, and thus an atactic structure. PNIPAM cleaved from uniform CNC/PNIPAM hybrids showed a similar lack of stereoregularity. By contrast, PNIPAM cleaved from patchy CNC/PNIPAM hybrids showed a different pattern in chemical shift, giving $m / r=74 / 26$. In order to determine if this observation was related to a templating effect similar to what has been observed using chitosan, ${ }^{40}$ free PNIPAM was synthesized in the presence of unmodified CNCs. This also displayed an upfield chemical shift of the - $\mathrm{CH}$ - group, but nevertheless indicated atactic PNIPAM. In order to determine if PNIPAM grown from NHSBiB-CNCs indeed had a higher $m / r$ ratio than PNIPAM grown from 2BPA-CNCs, the hybrids were analyzed by circular dichroism (CD) spectroscopy (Figure S9). In contrast to the other samples, the CD spectrum of patchy CNC/PNIPAM hybrids shows a broad absorption of righthanded circularly polarized light in the range of $325-500 \mathrm{~nm}$, which suggests a certain degree of optical activity due to an isotactic (helical) conformation. On the other hand, unmodified CNCs absorb left-handed circularly polarized light in the 200$400 \mathrm{~nm}$ region, likely as a result of left-handed chiral nematic arrangements. $^{41-44}$ The lower critical solution temperature (LCST) of tethered PNIPAM also depended on tether location (Figure S10). Overall, the results of ${ }^{1} \mathrm{H}$ NMR and $\mathrm{CD}$ spectroscopy provided evidence to suggest that the location of ATRP initiator sites on CNCs had an effect on the stereochemistry of surface-grown PNIPAM.

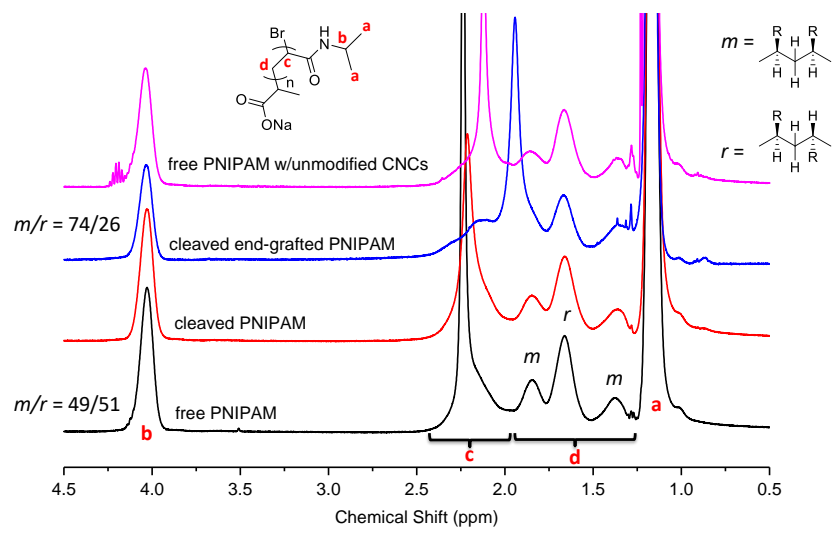

Figure 2. ${ }^{1} \mathrm{H}$ NMR spectra of PNIPAM cleaved from both uniform (red) and patchy (blue) CNC/PNIPAM hybrids and free PNIPAM synthesized via solution ATRP, either in the absence (black) or presence (magenta) of unmodified CNCs, in $\mathrm{CDCl}_{3}$.
The results of XPS, TGA and ${ }^{1} \mathrm{H}$ NMR spectroscopy provided only indirect evidence of the desired patchy CNC hybrid structures in comparison to uniformly modified CNCs. In order to directly visualize reducing end group-selective chemical modification of CNCs, sub-5 nm Au nanoparticles (AuNPs) stabilized by anionic surface charges were synthesized and electrostatically adsorbed to patchy CNC/PMETAC hybrids. Shown in Figure 3 is a typical cryo-electron micrograph of AuNP-labeled patchy CNC/PMETAC hybrids. The image reveals slightly aggregated $\mathrm{CNC}$ hybrid structures in which the AuNPs appear to be concentrated at the end groups containing tethered PMETAC chains. In some cases, both ends of CNCs appear to be functionalized, likely because the source CNCs also contained some portion of cellulose II crystals with antiparallel chain arrangements (vide supra). ${ }^{30}$ Since NHSactive esters could have also potentially reacted with CNC hydroxyl groups, although at a significantly lower rate, some degree of functionalization along the principal $\mathrm{CNC}$ axis was unavoidable. This will be remedied in our future work by ester group selective hydrolysis, which will preserve polymers tethered via amide bonds intact, i.e. at the reducing end groups. We also observed some CNCs that appeared either unfunctionalized with polymer tethers or unlabeled with AuNPs. It will be therefore critical in future work to optimize the reaction conditions, such that reducing end group functionalization is maximized to produce homogeneous samples. Nevertheless, Figure 3 clearly demonstrates the proofof-concept for the synthesis of "patchy" CNC hybrids. We further attempted to analyze AuNP-labeled uniform CNC/PMETAC hybrids, but the TEM images show only very few bound AuNPs (Figure S11), probably because of the low overall PMETAC content, as shown in Table 1.

In summary, we presented water tolerant reaction pathways toward both uniform and "patchy" CNC hybrids with a variety of tethered polymers via SI-ATRP. The stereochemistry of PNIPAM appeared to be affected by the precise location of ATRP initiating sites, perhaps related to the inherent righthanded chirality of CNCs. ${ }^{34-36}$ Moving forward, our objectives will be to optimize the reaction conditions such that reducing end group modification is maximized, graft hydrophobic polymers to create amphiphilic $\mathrm{CNC}$ hybrids and investigate the self-assembly of a library of patchy CNC hybrids in a variety of solvents. We anticipate the present work is the starting point for the development of both symmetric and asymmetric CNCs with end-tethered polymer chains as an experimental platform to study the self-assembly of end-functionalized anisotropic nanohybrids. $1,7,20$ 


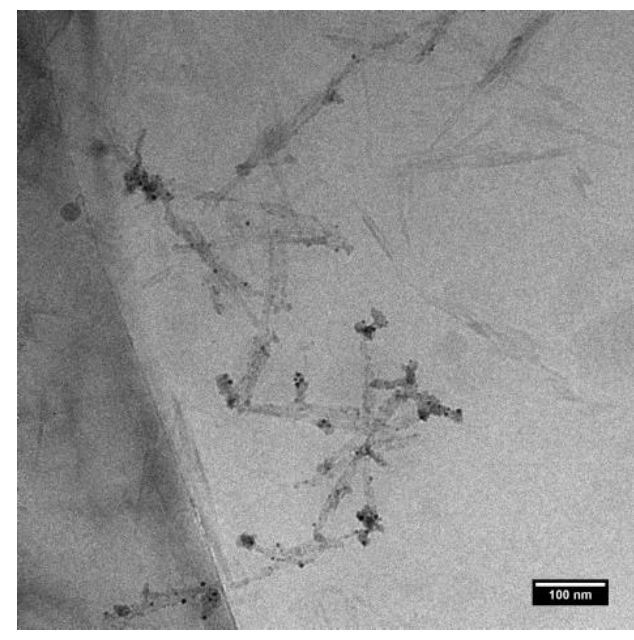

Figure 3. Cryo-EM micrograph of patchy CNC/PMETAC hybrids (CNC-RE-g-PMETAC) labeled with AuNPs.

\section{ASSOCIATED CONTENT}

\section{Supporting Information}

Experimental section including materials and methods, results of elemental analysis, Langmuir adsorption isotherms of Congo red dye, FTIR analysis, TGA of CNC/PSS and CNC/PMETAC hybrids, ${ }^{1} \mathrm{H}$ NMR spectra of cleaved PMETAC and PSS, CD spectra and LCST curves of CNC/PNIPAM hybrids.

The Supporting Information is available free of charge on the ACS Publications website.

\section{AUTHOR INFORMATION}

\section{Corresponding Author}

*Justin O. Zoppe, Adolphe Merkle Institute, University of Fribourg, CH-1700 Fribourg, Switzerland

Email: justin.zoppe@unifr.ch

\section{Present Addresses}

$\uparrow$ Adolphe Merkle Institute, University of Fribourg, CH-1700 Fribourg, Switzerland

\section{Author Contributions}

The manuscript was written through contributions of all authors. / All authors have given approval to the final version of the manuscript.

\section{Funding Sources}

Financial support from the Swiss National Science Foundation (SNSF) (Ambizione Grant no. PZ00P2_167900; J. Zoppe) and the EPFL Fellows Marie Curie COFUND program (EU FP7 grant agreement no. 291771; J. Zoppe) is gratefully acknowledged. C. Weder acknowledges financial support through the Army Research Office under Grant No. W911NF-15-1-0190.

\section{ACKNOWLEDGMENT}

The authors would like to thank Clémence Marie-Laure Piquart for assistance developing Fischer esterification protocols, Christoph Geers for helpful discussions, Pierre Mettraux for XPS analysis and Euro Solari for elemental analysis.

\section{ABBREVIATIONS}

\section{AuNPs gold nanoparticles}

2BPA 2-bromopropionic acid
CNCs cellulose nanocrystals

EDA ethylenediamine

EDC $\cdot \mathrm{HCl} N$-(3-dimethylaminopropyl)- $N$ '-ethylcarbodiimide hydrochloride

EISA evaporation-induced self-assembly

FRP free radical polymerization

LC liquid crystal

LCST lower critical solution temperature

METAC [2-(methacryloyloxy)ethyl]trimethylammonium chloride NHS-BiB 2-bromoisobutanoic acid N-hydroxysuccinimide ester NIPAM $N$-isopropylacrylamide

PMDETA $N, N, N^{\prime}, N^{\prime \prime}, N^{\prime \prime}$-pentamethyldiethylenetriamine

PMAA poly(methacrylic acid)

PMMA poly(methyl methacrylate)

RE reducing end groups

SI-ATRP surface-initiated atom transfer radical polymerization

SI-CRP surface-initiated controlled radical polymerization 4-SS sodium 4-vinylbenzenesulfonate

\section{REFERENCES}

(1) Lunn, D. J.; Finnegan, J. R.; Manners, I. Self-assembly of "patchy" nanoparticles: a versatile approach to functional hierarchical materials. Chem. Sci. 2015, 6 (7), 3663-3673.

(2) Walther, A.; Muller, A. H. E. Janus Particles: Synthesis, Self-Assembly, Physical Properties, and Applications. Chem. Rev. 2013, 113 (7), 5194-5261.

(3) Lee, S. W.; Mao, C. B.; Flynn, C. E.; Belcher, A. M. Ordering of Quantum Dots Using Genetically Engineered Viruses. Science 2002, 296 (5569), 892-895.

(4) Whaley, S. R.; English, D. S.; Hu, E. L.; Barbara, P. F.; Belcher, A. M. Selection of peptides with semiconductor binding specificity for directed nanocrystal assembly. Nature 2000, 405 (6787), 665-668.

(5) Rupar, P. A.; Chabanne, L.; Winnik, M. A.; Manners, I. Non-Centrosymmetric Cylindrical Micelles by Unidirectional Growth. Science 2012, 337 (6094), 559562.

(6) Nie, Z. H.; Fava, D.; Kumacheva, E.; Zou, S.; Walker, G. C.; Rubinstein, M. Self-assembly of metal-polymer analogues of amphiphilic triblock copolymers. Nat. Mater. 2007, 6 (8), 609-614.

(7) Glotzer, S. C.; Solomon, M. J. Anisotropy of building blocks and their assembly into complex structures. Nat. Mater. 2007, 6 (8), 557-562.

(8) Lagerwall, J. P. F.; Scalia, G. A new era for liquid crystal research: Applications of liquid crystals in soft matter nano-, bio- and microtechnology. Curr. Appl. Phys. 2012, 12 (6), 1387-1412.

(9) Dussi, S.; Dijkstra, M. Entropy-driven formation of chiral nematic phases by computer simulations. Nat. Commun. 2016, 7, 11175.

(10) Chung, W. J.; Oh, J. W.; Kwak, K.; Lee, B. Y.; Meyer, J.; Wang, E.; Hexemer, A.; Lee, S. W. Biomimetic selftemplating supramolecular structures. Nature 2011, 478 (7369), 364-368.

Barry, E.; Beller, D.; Dogic, Z. A model liquid crystalline system based on rodlike viruses with variable chirality and persistence length. Soft Matter 2009, 5 (13), 25632570 .

(12) Giraud-guille, M.-M. Cholesteric Twist of Collagen In Vivo and In Vitro. Mol. Cryst. Liq. Cryst. Inc. Nonlinear Opt. 1987, 153 (1), 15-30.

Revol, J. F.; Marchessault, R. H. In-Vitro Chiral Nematic Ordering of Chitin Crystallites. Int. J. Biol. Macromol. 1993, 15 (6), 329-335. 
Habibi, Y.; Lucia, L. A.; Rojas, O. J. Cellulose Nanocrystals: Chemistry, Self-Assembly, and Applications. Chem. Rev. 2010, 110 (6), 3479-3500.

Giese, M.; Blusch, L. K.; Khan, M. K.; MacLachlan, M. J. Functional Materials from Cellulose-Derived LiquidCrystal Templates. Angew. Chem. Int. Ed. (English) 2015, 54 (10), 2888-2910.

Azzam, F.; Heux, L.; Jean, B. Adjustment of the Chiral Nematic Phase Properties of Cellulose Nanocrystals by Polymer Grafting. Langmuir 2016, 32 (17), 4305-4312.

Yi, J.; Xu, Q. X.; Zhang, X. F.; Zhang, H. L. Chiralnematic self-ordering of rodlike cellulose nanocrystals grafted with poly(styrene) in both thermotropic and lyotropic states. Polymer 2008, 49 (20), 4406-4412.

Schutz, C.; Agthe, M.; Fall, A. B.; Gordeyeva, K.; Guccini, V.; Salajkova, M.; Plivelic, T. S.; Lagerwall, J. P. F.; Salazar-Alvarez, G.; Bergstrom, L. Rod Packing in Chiral Nematic Cellulose Nanocrystal Dispersions Studied by Small-Angle X-ray Scattering and Laser Diffraction. Langmuir 2015, 31 (23), 6507-6513.

Kim, N. H.; Imai, T.; Wada, M.; Sugiyama, J. Molecular directionality in cellulose polymorphs. Biomacromolecules 2006, 7 (1), 274-280.

He, L. L.; Zhang, L. X.; Ye, Y. S.; Liang, H. J. SolventInduced Self-Assembly of Polymer-Tethered Nanorods. J. Phys. Chem. B. 2010, 114 (21), 7189-7200.

Zoppe, J. O.; Cavusoglu Ataman, N.; Piotr, M.; Wang, J.; Moraes, J.; Klok, H.-A. Surface-Initiated Controlled Radical Polymerization: State-of-the-Art, Opportunities, and Challenges in Surface and Interface Engineering with Polymer Brushes. Chem. Rev. 2017, 117 (3), 11051318.

Lokanathan, A. R.; Nykanen, A.; Seitsonen, J.; Johansson, L. S.; Campbell, J.; Rojas, O. J.; Ikkala, O.; Laine, J. Cilia-Mimetic Hairy Surfaces Based on EndImmobilized Nanocellulose Colloidal Rods. Biomacromolecules 2013, 14 (8), 2807-2813.

Arcot, L. R.; Lundahl, M.; Rojas, O. J.; Laine, J. Asymmetric cellulose nanocrystals: thiolation of reducing end groups via NHS-EDC coupling. Cellulose 2014, 21 (6), 4209-4218.

Sipahi-Saglam, E.; Gelbrich, M.; Gruber, E. Topochemically modified cellulose. Cellulose 2003, 10 (3), 237-250.

Balamurugan, S. S.; Subramanian, B.; Bolivar, J. G.; McCarley, R. L. Aqueous-Based Initiator Attachment and ATRP Grafting of Polymer Brushes from Poly(methyl methacrylate) Substrates. Langmuir 2012, 28 (40), 14254-14260.

Boujemaoui, A.; Mongkhontreerat, S.; Malmstrom, E.; Carlmark, A. Preparation and characterization of functionalized cellulose nanocrystals. Carbohydr. Polym. 2015, 115, 457-464.

Braun, B.; Dorgan, J. R. Single-Step Method for the Isolation and Surface Functionalization of Cellulosic Nanowhiskers. Biomacromolecules 2009, 10 (2), 334341.

Beck, S.; Methot, M.; Bouchard, J. General procedure for determining cellulose nanocrystal sulfate half-ester content by conductometric titration. Cellulose 2015, 22 (1), 101-116.

Goodrich, J. D.; Winter, W. T. alpha-Chitin nanocrystals prepared from shrimp shells and their specific surface area measurement. Biomacromolecules 2007, 8 (1), 252257.

Reid, M. S.; Villalobos, M.; Cranston, E. D. Benchmarking Cellulose Nanocrystals: From the
Laboratory to Industrial Production. Langmuir 2017, 33 (7), 1583-1598.

Jiang, F.; Esker, A. R.; Roman, M. Acid-Catalyzed and Solvolytic Desulfation of H2SO4-Hydrolyzed Cellulose Nanocrystals. Langmuir 2010, 26 (23), 17919-17925.

Zoppe, J. O.; Habibi, Y.; Rojas, O. J.; Venditti, R. A.; Johansson, L.-S.; Efimenko, K.; Osterberg, M.; Laine, J. Poly(N-isopropylacrylamide) Brushes Grafted from Cellulose Nanocrystals via Surface-Initiated SingleElectron Transfer Living Radical Polymerization. Biomacromolecules 2010, 11 (10), 2683-2691.

Tang, W.; Kwak, Y.; Braunecker, W.; Tsarevsky, N. V.; Coote, M. L.; Matyjaszewski, K. Understanding atom transfer radical polymerization: Effect of ligand and initiator structures on the equilibrium constants. J. Am. Chem. Soc. 2008, 130 (32), 10702-10713.

Majoinen, J.; Haataja, J. S.; Appelhans, D.; Lederer, A.; Olszewska, A.; Seitsonen, J.; Aseyev, V.; Kontturi, E.; Rosilo, H.; Osterberg, M.et al. Supracolloidal Multivalent Interactions and Wrapping of Dendronized Glycopolymers on Native Cellulose Nanocrystals. J. Am. Chem. Soc. 2014, 136 (3), 866-869.

Majoinen, J.; Hassinen, J.; Haataja, J. S.; Rekola, H. T.; Kontturi, E.; Kostiainen, M. A.; Ras, R. H.; Torma, P.; Ikkala, O. Chiral Plasmonics Using Twisting along Cellulose Nanocrystals as a Template for Gold Nanoparticles. Adv. Mater. 2016, 28 (26), 5262-5267.

Usov, I.; Nystrom, G.; Adamcik, J.; Handschin, S.; Schutz, C.; Fall, A.; Bergstrom, L.; Mezzenga, R. Understanding nanocellulose chirality and structureproperties relationship at the single fibril level. Nat. Commun. 2015, 6, 7564.

Idota, N.; Nagase, K.; Tanaka, K.; Okano, T.; Annaka, M. Stereoregulation of Thermoresponsive Polymer Brushes by Surface-Initiated Living Radical Polymerization and the Effect of Tacticity on Surface Wettability. Langmuir 2010, 26 (23), 17781-17784.

Sato, M.; Kato, T.; Ohishi, T.; Ishige, R.; Ohta, N.; White, K. L.; Hirai, T.; Takahara, A. Precise Synthesis of Poly(methyl methacrylate) Brush with Well-Controlled Stereoregularity Using a Surface-Initiated Living Anionic Polymerization Method. Macromolecules 2016, 49 (6), 2071-2076.

Satoh, K.; Kamigaito, M. Stereospecific Living Radical Polymerization: Dual Control of Chain Length and Tacticity for Precision Polymer Synthesis. Chem. Rev. 2009, 109 (11), 5120-5156.

Kataoka, S.; Ando, T. Induced Optical-Activity of Acridine-Orange Bonded to Optically-Active Poly(Methacrylic Acid) by Radical Polymerization in the Presence of Chitosan. Polym. Commun. 1984, 25 (1), 24 26.

Therien-Aubin, H.; Lukach, A.; Pitch, N.; Kumacheva, E. Coassembly of Nanorods and Nanospheres in Suspensions and in Stratified Films. Angew. Chem. Int. Ed. (English) 2015, 54 (19), 5618-5622.

Araki, J.; Kuga, S. Effect of trace electrolyte on liquid crystal type of cellulose microcrystals. Langmuir 2001, 17 (15), 4493-4496.

Risteen, B. E.; Blake, A.; McBride, M. A.; Rosu, C.; Park, J. O.; Srinivasarao, M.; Russo, P. S.; Reichmanis, E. Enhanced Alignment of Water-Soluble Polythiophene Using Cellulose Nanocrystals as a Liquid Crystal Template. Biomacromolecules 2017, 18 (5), 1556-1562. Wang, P. X.; Hamad, W. Y.; MacLachlan, M. J. Structure and transformation of tactoids in cellulose nanocrystal suspensions. Nat. Commun. 2016, 7. 

TOC Graphic
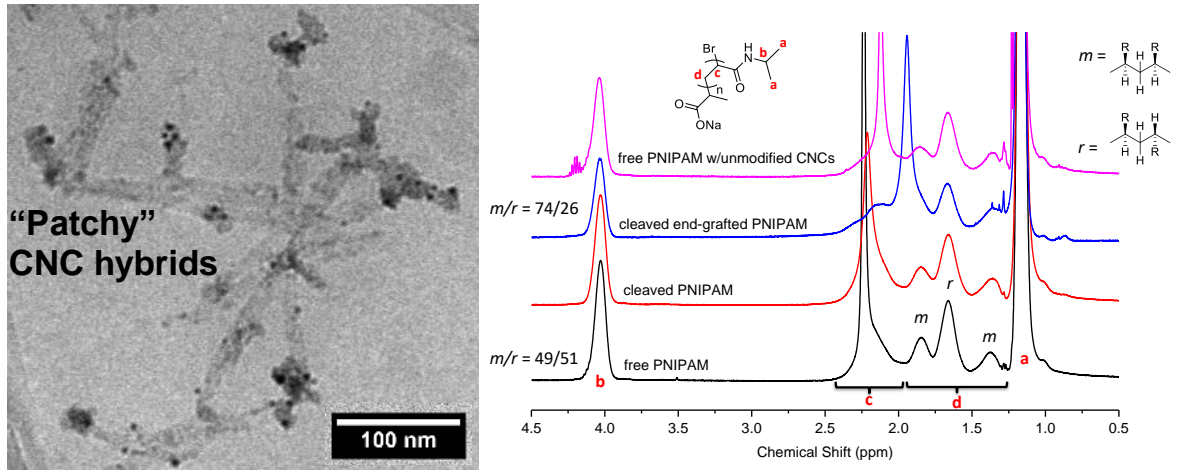